\title{
Source Analysis and Selection Using Block Term Decomposition in Atrial Fibrillation
}

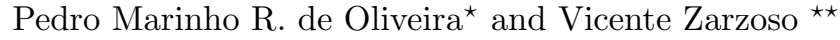 \\ Université Côte d'Azur, CNRS, I3S Laboratory, \\ CS 40121, 06903 Sophia Antipolis Cedex, France \\ \{marinho, zarzoso\}@i3s. unice.fr
}

\begin{abstract}
Atrial fibrillation (AF) is the most common sustained cardiac arrhythmia in clinical practice, and is becoming a major public health concern. To better understand the mechanisms of this arrhythmia an accurate analysis of the atrial activity (AA) signal in electrocardiogram (ECG) recordings is necessary. The block term decomposition (BTD), a tensor factorization technique, has been recently proposed as a tool to extract the AA in ECG signals using a blind source separation (BSS) approach. This paper makes a deep analysis of the sources estimated by BTD, showing that the classical method to select the atrial source among the other sources may not work in some cases, even for the matrix-based methods. In this context, we propose two new automated methods to select the atrial source by considering another novel parameter. Experimental results on ten patients show the validity of the proposed methods.
\end{abstract}

Keywords: Atrial Source Selection, Block Term Decomposition, Atrial Fibrillation, Blind Source Separation

\section{Introduction}

Atrial fibrillation (AF) is the most common sustained cardiac arrhythmia in clinical practice, responsible for up to $25 \%$ of strokes and $1 / 3$ of the hospitalizations due to cardiac related disturbances [1]. This arrhythmia is becoming a major public health concern, since about 160000 new AF cases are discovered every year only in USA, with similar numbers in European countries. This makes $\mathrm{AF}$ an increasingly prevalent disease that could become a new epidemic over the years [2]. The mechanisms of this supraventricular arrhythmia are not completely understood, making AF a challenging cardiac condition, considered as the last great frontier of cardiac electrophysiology. During AF, electrical impulses typically generated around the pulmonary veins propagate in a chaotic and irregular way across the atria, replacing the $\mathrm{P}$ wave, that corresponds to a normal atrial activation, by low-amplitude fibrillatory waves, or f-waves. The f-waves

\footnotetext{
* Pedro Marinho is funded by a PhD scholarship from the IT Doctoral School of the Université Côte d'Azur.

** Vicente Zarzoso is a member of the Institut Universitaire de France.
} 
are present through all the electrocardiogram (ECG) recording, but masked by the QRS complex of ventricular activity (VA) in each heartbeat.

To better understand the mechanisms of AF, it is necessary an accurate analysis of the atrial activity (AA), specifically, the $\mathrm{f}$ waves. A noninvasive analysis can be made by extracting the AA from the cardiac signals recorded by the standard 12-lead ECG using matrix decompositions techniques for blind source separation (BSS), such as principal component analysis (PCA) and independent component analysis (ICA) [3-5]. This matrix decomposition approach has proven to be useful for AA extraction. However, it has some limitations, since constraints need to be imposed to guarantee the uniqueness of such decompositions, e.g., orthogonality or statistical independence between the sources. Although mathematically coherent, such constraints may lack physiological grounds.

In order to overcome these limitations, a tensor approach has recently been proposed to analyze AF signals [6-9]. As compared to matrix techniques, tensor decompositions present some remarkable features such as essential uniqueness with practically minimal or no constraints. The block term decomposition (BTD) proposed in [12] suits the characteristics of AA in an AF signal, since atrial signals can be approximated by all-pole models and mapped onto Hankel matrices with rank equal to the number of poles [9]. These Hankel matrices that contain the ECG data are stacked in the third dimension of a $3^{\text {rd }}$-order tensor, and then processed by BTD. Previous experimental results in synthetic and real ECG data showed the potential superiority of BTD as compared to matrix decompositions for short ECG recordings [6-8].

The success of the BSS approach to AA extraction depends on the accurate identification of the atrial signal among the estimated sources. The classical method for atrial source selection consists in selecting the source with the highest spectral concentration (SC) among the sources whose dominant frequency (DF) lies between 3 and $9 \mathrm{~Hz}[3,4]$. The present work makes a deep analysis in the sources estimated by BTD, showing that the classical method may not work in some cases, even when the matrix-based approach is used. Taking this into account, a new parameter to improve the performance of the classical method is proposed. This parameter consists in analyzing the power of the source contribution to the lead V1, a lead that significantly reflects AA. Also, a new automated method for atrial source selection is proposed, using the proposed parameter and another one based on the kurtosis of the signal in the frequency domain. Experimental results using ten patients with persistent $\mathrm{AF}$ evaluate the proposed methods, showing their better performance in selecting the atrial source among the sources estimated by BTD and two matrix-based methods previously proposed in literature for AA extraction: RobustICA-f [10] and PCA [11]. It is also shown that BTD can provide a better estimation of the AA signal, outperforming the matrix-based techniques in most cases.

The rest of this paper is organized as follows. Section 2 introduces the notation used in the work. Section 3 recalls the BTD as a tensor approach to solve BSS problems, while Section 4 discusses the estimated sources and the atrial source selection methods. Section 5 presents the experimental results and, fi- 
nally, Section 6 formulates the conclusion of this work, as well as the prospects for future works.

\section{Notations}

Scalars, vectors, matrices and tensors are represented by lower-case $(a, b$, $c, \ldots)$, boldface lower-case $(\mathbf{a}, \mathbf{b}, \mathbf{c}, \ldots)$, boldface capital $(\mathbf{A}, \mathbf{B}, \mathbf{C}, \ldots)$ and calligraphic $(\mathcal{A}, \mathcal{B}, \mathcal{C}, \ldots)$ letters, respectively.

The transpose is represented by $(\cdot)^{T}$, symbol $\|\cdot\|$ represents the $l_{2}$-norm and $\circ$ represents the outer product. The operator $\operatorname{diag}(\cdot)$ builds a diagonal matrix by placing its arguments along the diagonal. Given a $3^{\text {rd }}$-order tensor $\mathcal{A} \in \mathbb{C}^{I_{1} \times I_{2} \times I_{3}}$, with scalars $a_{i_{1}, i_{2}, i_{3}}$, its frontal slices are represented by $\mathbf{A}_{. . i_{3}} \in \mathbb{C}^{I_{1} \times I_{2}}$. Given a matrix $\mathbf{A} \in \mathbb{C}^{I_{1} \times I_{2}}$, with scalars $a_{i_{1}, i_{2}}$, its $i_{1}^{\text {th }}$ row and the $i_{2}^{\text {th }}$ column are represented by $\mathbf{a}_{i_{1}}$, and $\mathbf{a}_{i_{2}}$, respectively.

\section{Block Term Decomposition}

The BTD of an arbitrary $3^{\text {rd }}$-order tensor $\mathcal{T} \in \mathbb{R}^{I_{1} \times I_{2} \times I_{3}}$ is written as

$$
\mathcal{T}=\sum_{r=1}^{R} \mathbf{E}_{r} \circ \mathbf{c}_{r}
$$

with $\mathbf{c}_{r} \in \mathbb{R}^{I_{3}}$. Matrix $\mathbf{E}_{r} \in \mathbb{R}^{I_{1} \times I_{2}}$ has rank $L_{r}$ and admits a decomposition $\mathbf{E}_{r}=\mathbf{A}_{r} \mathbf{B}_{r}^{T}$, where $\mathbf{A}_{r} \in \mathbb{R}^{I_{1} \times L_{r}}$ and $\mathbf{B}_{r} \in \mathbb{R}^{I_{2} \times L_{r}}$ have rank $L_{r}$. We may then rewrite (1) as

$$
\mathcal{T}=\sum_{r=1}^{R}\left(\mathbf{A}_{r} \mathbf{B}_{r}^{T}\right) \circ \mathbf{c}_{r}
$$

One can see that the BTD is a decomposition of $\mathcal{T}$ in multilinear rank$\left(L_{r}, L_{r}, 1\right)$ terms. If the matrix factors $\mathbf{A}=\left[\mathbf{A}_{1} \mathbf{A}_{2} \ldots \mathbf{A}_{R}\right] \in \mathbb{R}^{I_{1} \times \sum_{r=1}^{R} L_{r}}$ and $\mathbf{B}=\left[\mathbf{B}_{1} \mathbf{B}_{2} \ldots \mathbf{B}_{R}\right] \in \mathbb{R}^{I_{2} \times \sum_{r=1}^{R} L_{r}}$ are full-column rank, which requires that $\sum_{r=1}^{R} L_{r} \leq I_{1}, I_{2}$, and $\mathbf{C}=\left[\mathbf{c}_{1} \mathbf{c}_{2} \ldots \mathbf{c}_{R}\right] \in \mathbb{R}^{I_{3} \times R}$ does not contain proportional columns, then the BTD is essentially unique [12, Theorem 2.2]. Milder uniqueness conditions are presented in [12].

The ECG data matrix, with $K$ leads and $N$ samples, can be modeled as

$$
\mathbf{Y}=\mathbf{M S} \in \mathbb{R}^{K \times N},
$$

where $\mathbf{M} \in \mathbb{R}^{K \times R}$ is the mixing matrix, modelling the propagation of the cardiac electrical sources from the heart to the body surface, $\mathbf{S} \in \mathbb{R}^{R \times N}$ is the source matrix that contains the atrial, ventricular and noise sources, and $R$ is the number of sources [5]. The AA extraction in an AF ECG recording can be seen as a BSS problem, since the only data observed is matrix $\mathbf{Y}$, and we aim to estimate $\mathbf{M}$ and $\mathbf{S}$ from it. In [12], the BTD is proposed as a solution of a BSS problem like (3), but does not deal with the AA extraction specifically. 
The idea to obtain a tensor from $\mathbf{Y}$ is to map its $k^{\text {th }}$ row onto a Hankel matrix $\mathbf{H}_{\mathbf{Y}}^{(k)} \in \mathbb{R}^{I \times J}$, where $I=J=\frac{N+1}{2}$ if $N$ is odd or $I=\frac{N}{2}$ and $J=\frac{N}{2}+1$ if $N$ is even, with

$$
\left[\mathbf{H}_{\mathbf{Y}}^{(k)}\right]_{i, j} \triangleq y_{k, i+j-1}
$$

where $i=1, \ldots, I, j=1, \ldots, J$, and $k=1, \ldots, K$. Next, the tensor is built by stacking each Hankel matrix along the third dimension (as frontal slices) of a $3^{\text {rd }}$-order tensor $\mathcal{Y} \in \mathbb{R}^{I \times J \times K}$, that is

$$
\mathbf{Y}_{. . k}=\mathbf{H}_{\mathbf{Y}}^{(k)}
$$

The $k^{\text {th }}$ matrix slice of the tensor $\mathcal{Y}$ can be represented as

$$
\mathbf{Y}_{. . k}=\sum_{r=1}^{R} m_{k, r} \mathbf{H}_{\mathbf{S}}^{(r)}
$$

where $\mathbf{H}_{\mathbf{S}}^{(r)} \in \mathbb{R}^{I \times J}$ is a Hankel matrix built from the $r^{\text {th }}$ row of $\mathbf{S}$. We can see that for each $r$, the outer product between matrix $\mathbf{H}_{\mathbf{S}}^{(r)}$ and the $r^{\text {th }}$ column of $\mathbf{M}$, i.e., $\mathbf{m}_{. r}$, is being performed. This way, the tensor $\mathcal{Y}$ can be written as

$$
\mathcal{Y}=\sum_{r=1}^{R} \mathbf{H}_{\mathbf{S}}^{(r)} \circ \mathbf{m}_{\cdot r}
$$

Comparing Equation (1) with (7), we can conclude that the tensor ECG data follows a BTD tensor model.

During AF, the AA presents certain harmonicity. Hence, atrial sources can plausibly be represented by the exponential (or all-pole) model as

$$
s_{r, n}=\sum_{\ell=1}^{L_{r}} \lambda_{\ell, r} z_{\ell, r}^{n-1}
$$

where $n=1, \ldots, N, r=1, . ., R, L_{r}$ is the number of exponential terms, $z_{\ell, r}$ is the $\ell^{\text {th }}$ pole of the $r^{\text {th }}$ source, and $\lambda_{\ell, r}$ is the scaling coefficient [6-9]. This way, their associated Hankel matrix accepts the Vandermonde decomposition as in [13].

\section{Atrial Source Selection}

\subsection{Classical Method}

To select the AA signal or the source with the most significant AA activity, the classical method considers two parameters. The first one is the DF, that is, the position of the peak frequency in the power spectral density, since the AA during AF typically has a peak between 3 and $9 \mathrm{~Hz}$. The second parameter, 
called SC, is the relative amount of energy around the DF, and it is calculated as:

$$
S C=\frac{\sum_{0.82 f_{p}}^{1.17 f_{p}} P_{A A}\left(f_{i}\right)}{\sum_{0}^{F_{s} / 2} P_{A A}\left(f_{i}\right)},
$$

where $f_{p}$ is the value of DF, $F_{S}$ is the sampling frequency and $P_{A A}$ is the power spectrum of the AA signal, estimated as in [4]. In this work, the $\mathrm{SC}$ is calculated over the first harmonic (fundamental frequency) only.

The classical method of atrial source selection makes the assumption that the atrial source is concentrated in a single source only. This method consists of the following steps:

1. Select all the estimated sources with DF between 3 and $9 \mathrm{~Hz}$. We refer to sources fulfilling this condition as potential atrial sources.

2. Select the potential atrial source with the highest SC.

\subsection{Proposed Method 1}

In the literature, the classical automated method described above has been used to detect the atrial source among the other estimated sources. However, in some cases, this method may not precisely select the atrial source, as will be illustrated later in this work. In Figures 3 and 4, for example, the atrial source does not correspond to the potential source with the highest SC, despite the fact that they have close values of SC at very close DF positions.

Aiming at a better estimation of the AA signal, this paper proposes two new parameters. The first new parameter is the power contribution to the recording, which is given by

$$
P(r)=\frac{1}{N}\left\|m_{r}^{(V 1)} \mathbf{s}_{r .}\right\|^{2},
$$

in $\mathrm{mV}^{2}$, where $m_{r}^{(V 1)}$ is the contribution of the $r^{\text {th }}$ source to lead V1 (given by the corresponding element of the estimated mixing matrix) and $\mathbf{s}_{r}$. is the $r^{\text {th }}$ source, corresponding to the $r^{\text {th }}$ row of matrix $\mathbf{S}$ in Equation (3). Using the power contribution to the recording as a new parameter the classical method becomes:

1. Select all the estimated sources with DF between 3 and $9 \mathrm{~Hz}$ (potential atrial sources).

2. Select all the potential atrial sources with power contribution higher than $10^{-4} \mathrm{mV}^{2}$. We refer to this subset of sources as likely atrial sources.

3. Select the likely atrial source with the highest SC.

Selecting the sources with power contribution higher than $10^{-4} \mathrm{mV}^{2}$ is needed in order to eliminate all sources that may present AA-like signature but are actually too weak to represent AA components. This threshold is chosen based on initial experiments that showed that sources with power contribution lower than $10^{-4} \mathrm{mV}^{2}$ do not present any significant contribution to the original signal. The power contribution is calculated in lead V1 due to the fact that this lead typically reflects AA best in AF ECGs, as its exploring electrode lies close to the right atrium. 


\subsection{Proposed Method 2}

In order to better select the source with the most significant AA content among the other estimated sources, a new automated method is now proposed. The first two steps of this method are the same as those of the proposed method introduced in the previous subsection. The third and last step of this new method is to compute the kurtosis, denoted $K$, of the signal in the frequency domain acquired by a 4096-point FFT (the second new parameter). As in [10], we use the general expression of kurtosis valid for non-circular complex data. The likely atrial source with the highest kurtosis is related as the atrial source.

In the experiments below, it will be shown that selecting the source with the highest kurtosis provides a better performance than selecting the source with the highest SC. A possible explanation is that kurtosis is computed from the whole signal, while SC is only computed around the DF. Recall that AA in AF is typically a harmonic signal, characterized by a sparse frequency spectrum with few values significantly different from zero. Kurtosis is a measure of peakedness and sparsity of a distribution and, when computed in the frequency domain, it naturally provides a quantitative measure of harmonicity of the signal. Also, kurtosis is parameter free, whereas SC depends on the DF and the definition of a suitable interval for interpretation.

\section{Experimental Results}

\subsection{Real AF ECG Data and Preprocessing}

The recordings used in our experiments belong to a database provided by the Cardiology Department of the Princess Grace Hospital Center, Monaco. These recordings were acquired at a $977 \mathrm{~Hz}$ sampling rate and preprocessed by a zero-phase forward-backward type-II Chebyshev bandpass filter with cutoff frequencies of 0.5 and $40 \mathrm{~Hz}$, to suppress high-frequency noise and baseline wandering. To analyze the potential atrial sources, we consider a randomly selected heartbeat (QRS-T complex + TQ segment) of a standard 12-lead ECG recording from a persistent AF patient. A single-beat segment of this patient is shown in Figure 1, where we can see the TQ interval just after the QRS-T complex in lead II. The beat from this patient used to analyze the potential atrial sources is chosen randomly and has 1300 samples.

To asses the atrial selection methods, a population of 10 patients with persistent AF is used in the same way previously described. Similarly, one beat from each of the ten patients is chosen randomly to evaluate atrial source selection performance. The lengths of the chosen beats is between 1000 and 1400 samples (1.02 and 1.43 seconds, respectively). Due to lack of space, the potential source analysis of all ten patients is not reported in this paper. So only the first patient of the observed population was chosen for source analysis.

\subsection{BTD Setup}

The BTD is implemented using the non-linear least squares (NLS) method available in Tensorlab MATLAB toolbox [14] choosing $R=12$ and $L_{r}=95$, 


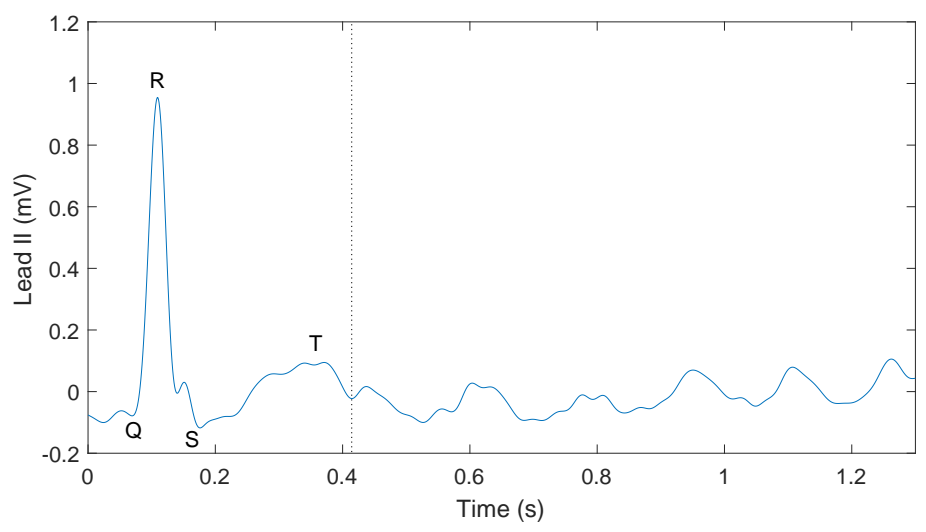

Fig. 1: A single-beat segment of an AF ECG recording of one patient in lead II. The vertical line marks the location of the T-wave offset.

for $r=1,2, \ldots, 12$. This choice is made based on the work [9], which showed that these values provided good results for the heartbeat with the largest TQ segment of one of the patients in the present observed population. The tolerance threshold for convergence is set to $10^{-9}$ and the maximum number of iterations is set to 1000 . BTD is known to be dependent on a suitable initialization of its factors. The experiments reported in this section evaluate the influence of BTD factors initialization on source estimation performance and atrial source selection. Ten Monte Carlo runs, with normalized Gaussian random initialization for the matrix and vector factors at each run, are used to analyze the potential atrial sources found by BTD and compare them with the ones found by the matrix-based methods PCA and RobustICA-f. All the beats are downsampled by a factor of two, since the $3^{\text {rd }}$-order tensor built from the original 12-lead ECG beat poses some difficulties to Tensorlab.

\subsection{Potential Atrial Source Analysis}

For the observed patient used to analyze the potential atrial sources, PCA found 6 potential sources, RobustICA-f found 5 potential sources and BTD found a mean of 7.2 potential sources. In 7 out of the 10 independent runs, the BTD found more potential sources than the matrix-based methods, reflecting the ability of the tensor technique to perform undertermined source separation [12]. Finding several potential atrial sources is interesting, since it increases the possibility of finding some features that, although weakly contributing to the AA, may provide useful physiological and clinical information about the arrhythmia. In this work, however, we assume as in the previous literature of this topic that all AA can be represented by a single source, and we leave the multiple source hypothesis for further works. Due to the lack of space and for the sake of clarity, only two potential atrial sources for PCA, RobustICA-f and BTD are shown 

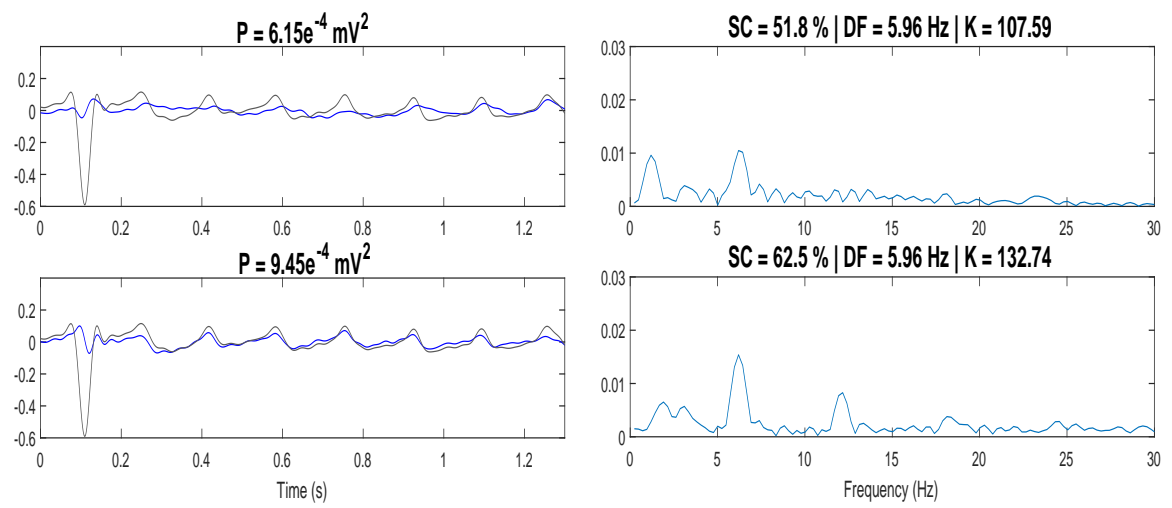

Fig. 2: Potential atrial sources contribution to lead V1 estimated by PCA. Left: time domain (in $\mathrm{mV}$ ). Right: frequency domain (in $\mathrm{mV} / \sqrt{\mathrm{Hz}}$ ).

in Figures 2-4. The other sources were disregarded for presenting a very weak power contribution.

Looking at Figure 2, we can see that the atrial source estimated by PCA (located in the second row) has SC equal to $62.5 \%$, while looking at Figure 3, the estimated atrial source by RobustICA-f (located in the second row) has SC equal to $68.3 \%$. For BTD, 8 out of the 10 independent runs estimated an atrial source with higher SC than PCA and 6 with higher SC than both matrix-based methods, giving an average SC over the 10 runs equal to $67.8 \%$. Figure 4 shows the results for a particular initialization of $\mathrm{BTD}$, where the estimated atrial source (located in the second row) has SC equal to 76.5\%. The DF position of both PCA and RobustICA-f are located at $5.96 \mathrm{~Hz}$, while in BTD it lies between 5.72 and $5.96 \mathrm{~Hz}$. For comparison, the DF position obtained from an electrogram simultaneously acquired by a catheter located in the left atrial appendage of the same patient, is equal to $4.77 \mathrm{~Hz}$.

\subsection{Atrial Source Selection}

As ground truth, the sources were visually analyzed in time and frequency domain with guidance of the parameters previously described. The source with the strongest representation of AA content was taken as the atrial source.

The classical method and the two proposed methods of atrial source selection were assessed in 10 segments of 10 different patients, as previously explained. From a total of 120 runs for the 10 patients (100 for BTD, 10 for PCA and 10 for RobustICA-f) the classical method succeeded only in $45.8 \%$ of runs. Applying the first proposed method, the index of success increases to $75 \%$, while the second proposed technique succeeds in $83.7 \%$ of the trials. It should be mentioned that in $35.8 \%$ of the trials, both the classical and the second proposed method were able to select the source with most AA content. Also, in $12.5 \%$ of trials none of the methods were able to select the AA signal. This means that the existing 

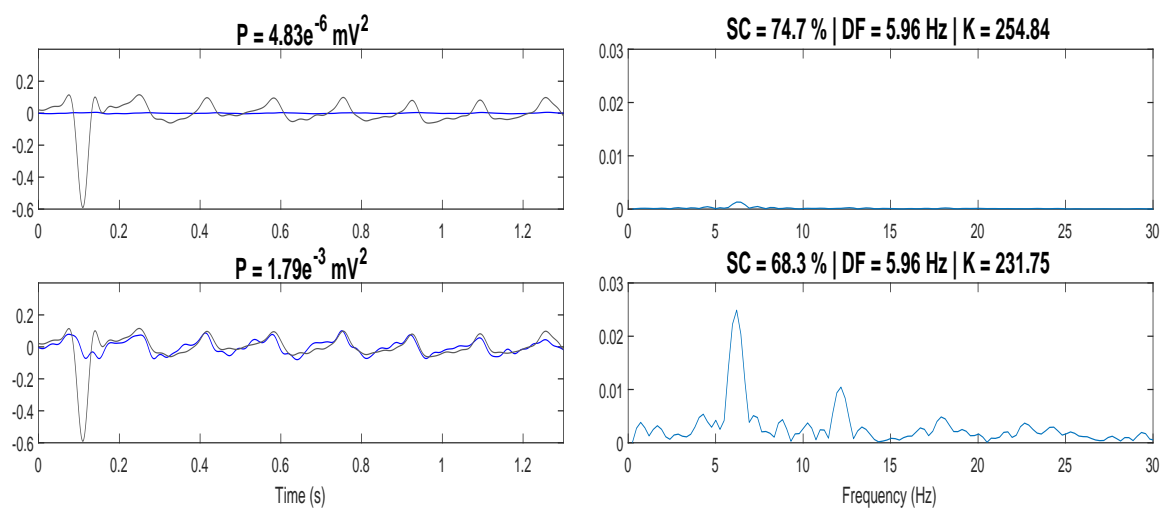

Fig. 3: Potential atrial sources contribution to lead V1 estimated by RobustICAf. Left: time domain (in $\mathrm{mV}$ ). Right: frequency domain (in $\mathrm{mV} / \sqrt{\mathrm{Hz}}$ ).
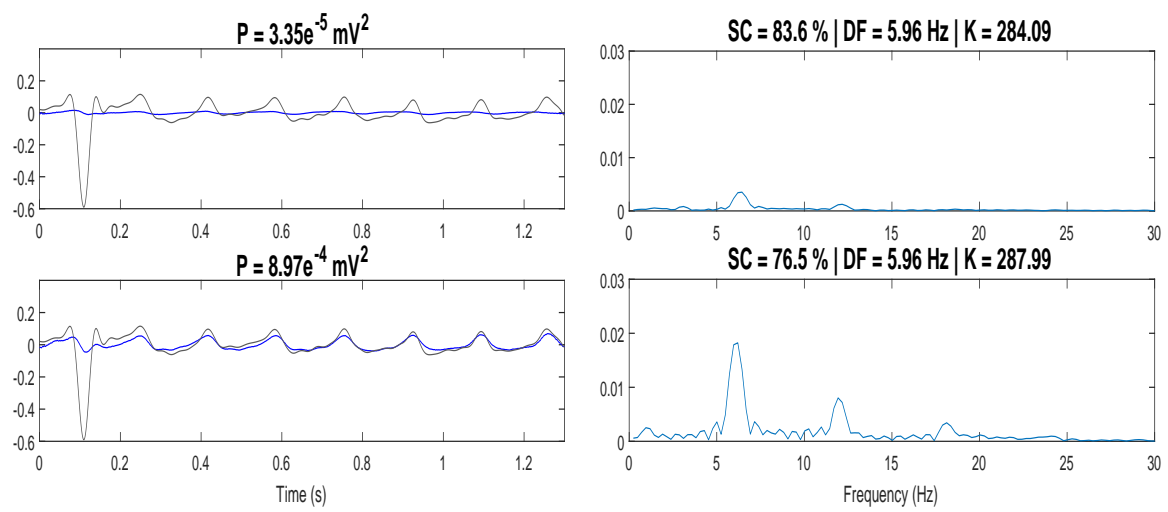

Fig. 4: Potential atrial sources contribution to lead V1 estimated by BTD for a single run. Left: time domain (in $\mathrm{mV}$ ). Right: frequency domain (in $\mathrm{mV} / \sqrt{\mathrm{Hz}}$ ).

methods are suboptimal regarding the AA source selection. However, from the reported experiments, it is believed that a balanced combination between power contribution and kurtosis may lead to an optimal or at least a better method.

\section{Conclusions}

The present work has analyzed the potential atrial sources estimated by BTD, showing its satisfactory performance for most initializations in the tested database. We have shown that the classical method of atrial source selection may not work in some cases, and we have proposed two new automated methods that better select the atrial source among the other potential sources. These methods have been validated in experimental results not only for BTD but also for the matrix-based methods PCA and RobustICA-f in a population of 10 patients 
with persistent AF. In future works, we aim to assess the proposed methods in a larger database and along consecutive time segments of each patient to analyze intra-patient (temporal) stability.

\section{References}

1. C. T. January, L. S. Wann, J. S. Alpert, H. Calkins, J. C. Cleveland, J. E. Cigarroa, J. B. Conti, et al., "2014 AHA/ACC/HRS guideline for the management of patients with atrial fibrillation: A report of the American College of Cardiology/American Heart Association Task Force on practice guidelines and the Heart Rhythm Society", Circulation, vol. 64, no. 21, pp. 2246-2280, Dec. 2014.

2. L. Mainardi, L. Sörnmo, S. Cerutti, "Understanding Atrial Fibrilation: The Signal Processing Contribution", Synthesis Lectures on Biomedical Engineering, Morgan \& Claypool Publishers, 2008.

3. J. J. Rieta, F. Castells, C. Sánchez, V. Zarzoso, and J. Millet, "Atrial activity extraction for atrial fibrillation analysis using blind source separation", IEEE Transactions on Biomedical Engineering, vol. 51, no. 7, pp. 1176-1186, Jul. 2004.

4. F. Castells, J. J. Rieta, J. Millet, and V. Zarzoso, "Spatiotemporal blind source separation approach to atrial activity estimation in atrial tachyarrhythmias", IEEE Transactions on Biomedical Engineering, vol. 52, no. 2, pp. 258-267, Feb. 2005.

5. V. Zarzoso, "Extraction of ECG characteristics using source separation techniques: exploiting statistical independence and beyond", Advanced Biosignal Processing, A. Naït-Ali, Ed. Berlin, Heidelberg: Springer Verlag, 2009, ch. 2, pp. 15-47.

6. L. N. Ribeiro, A. R. Hidalgo-Muñoz, and V. Zarzoso, "Atrial signal extraction in atrial fibrillation electrocardiograms using a tensor decomposition approach", in Proc. EMBC-2015, 37th Annual International Conference of the IEEE Engineering in Medicine and Biology Society, Milan, Italy, Aug. 25-29, 2015, pp. 6987-6990.

7. L. N. Ribeiro, A. R. Hidalgo-Muñoz, G. Favier, J. C. M. Mota, A. L. F. de Almeida, and V. Zarzoso, "A tensor decomposition approach to noninvasive atrial activity extraction in atrial fibrillation ECG", in Proc. EUSIPCO-2015, XXIII European Signal Processing Conference, Nice, France, Aug. 31-Sept. 4, 2015, pp. 2576-2580.

8. L. N. Ribeiro, A. L. F. de Almeida, and V. Zarzoso, "Enhanced block term decomposition for atrial activity extraction in atrial fibrillation ECG", in Proc. SAM2016, 9th IEEE Sensor Array and Multichannel Signal Processing Workshop, Rio de Janeiro, Brazil, July 10-13, 2016.

9. V. Zarzoso, "Parameter estimation in block term decomposition for noninvasive atrial fibrillation analysis", in Proc. CAMSAP-2017, IEEE Int. Work. on Comp. Advances in Multi-Sensor Adapt. Proc., Curaçao, Dutch Antilles, Dec. 10-13, 2017.

10. V. Zarzoso and P. Comon, "Robust independent component analysis by iterative maximization of the kurtosis contrast with algebraic optimal step size", IEEE Transactions on Neural Networks, vol. 21, no. 2, pp. 248-261, 2010.

11. I. Jolliffe, Principal Component Analysis, Wiley Online Library, 2005.

12. L. De Lathauwer, "Blind separation of exponential polynomials and the decomposition of a tensor in rank- $\left(l_{r}, l_{r}, 1\right)$ terms", SIAM Journal on Matrix Analysis and Applications, vol. 32, no. 4, pp. 1451-1474, 2011.

13. D. L. Boley, F. T. Luk, and D. Vandevoorde, "Vandermonde factorization of a Hankel matrix", in Proc. of the Work. on Sci. Comp., Hong Kong, March 1997.

14. N. Vervliet, O. Debals, L. Sorber, M. Van Barel, and L. De Lathauwer, Tensorlab 3.0, Available online, Mar. 2016. URL: https://www.tensorlab.net/ 